\title{
Study on the Extraction of Polyphenols from Blueberry Leaves and their antioxidant properties
}

\author{
Fenghong Liu ${ }^{1 *}$, Xianhao Cheng ${ }^{2 *}$,Wenguang Liu ${ }^{1}$, Jing Miu $^{3}$, Jiahui Wang ${ }^{1}$, Xiaotong Cui ${ }^{1}$, Xiaoshi lu ${ }^{1}$ \\ ${ }^{1}$ College of Chemical and Biological Engineering, QILU Institute of Technology, Jinan, Shandong 250200. \\ ${ }^{2}$ College of Agriculture, Ludong University, Yantai, Shandong, 264001. \\ ${ }^{3}$ College of Life Sciences, Ludong University, Yantai, Shandong, 264001.
}

\begin{abstract}
Blueberry leaf as materials, ethanol ultrasonic assisted extraction of blueberry polyphenols, ethanol concentration and solid-liquid ratio on blueberry ultrasonic time leaves influence the yield of polyphenols were researched, and the orthogonal experiment to optimize the ethanol ultrasonic assisted extraction technological conditions of blueberry leaf polyphenol polyphenols was used. The results showed that the optimal extraction conditions of polyphenols from blueberry leaves were as follows, $9.06 \%$ with 4 min ultrasonic time, $80 \%$ ethanol concentration and $1: 45(\mathrm{~g} / \mathrm{mL})$ ratio. Ultrasonic assisted extraction is a convenient and high yield method, which is suitable for the extraction of polyphenols from blueberry leaves.
\end{abstract}

\section{Introduction}

Blueberry leaves contain polysaccharides, polyphenols, amino acids, fatty acids, crude protein, crude fat, crude fiber, organic acids, vitamins, sugars and a large number of polyphenols. Polyphenol, also known as tannins and casuarinin, is a kind of mixture of secondary metabolites widely existing in plants. They are the general names of plant components with multiple hydroxy phenols in molecules ${ }^{[1]}$.Studies such as Li showed that blueberry leaf polyphenols could effectively prolong the shelf life of perch during cold storage, which is an effective food biological preservative ${ }^{[2]}$. In addition, plant polyphenols have strong antioxidant, scavenging free radicals, anticancer, anti-aging, antiviral and other effects, known as 'seventh nutrients' ${ }^{[3]}$.

However, in blueberry machining processing, blueberry leaves are often discarded as waste, resulting in huge waste of resources. Turhan et al. ${ }^{[4]}$ confirmed that the polyphenol content in blueberry fruits was significantly lower than that in blueberry leaves, and the antioxidant activity and free radical scavenging ability of blueberry leaves were stronger than those of blueberry fruits.

In the paper, blueberry leaf as materials, ethanol ultrasonic assisted extraction of blueberry polyphenols, ethanol concentration and solid-liquid ratio on blueberry research ultrasonic time leaves influence the yield of polyphenols, and using the orthogonal experiment to optimize the ethanol ultrasonic assisted extraction technological conditions of blueberry leaf polyphenol polyphenols.

\section{Materials and methods}

\subsection{Materials}

Blueberry leaf (cultivar 'Lanfeng' )

\subsection{Methods}

\subsubsection{Blueberry pretreatment}

A certain amount of blueberry leaves was taken to dry at $50^{\circ} \mathrm{C}$, and screen after crushing 100 mesh sieve.

\subsubsection{Standard curve drawing}

The 250mg gallic acid standard was weighed, and the volume was fixed to $250 \mathrm{~mL}$ volumetric flask with deionized water. The standard solution of $1 \mathrm{mg} / \mathrm{mL}$ was prepared, and $5 \mathrm{~mL}$ was removed from the standard solution of $1 \mathrm{mg} / \mathrm{mL}$. The volume was fixed to $100 \mathrm{~mL}$ volumetric flask, and the standard solution of $50 \mu \mathrm{g} / \mathrm{mL}$ was prepared. Accurately absorb 0, 0.5, 1.0, 1.5, 2.0, $2.5,3.0,3.5 \mathrm{~mL}$ standard solution in $25 \mathrm{~mL}$ volumetric flask, add $1 \mathrm{~mL}$ Folin-Ciocalteu reagent $0 \sim 8 \mathrm{~min}$, add $3 \mathrm{~mL} 10 \%$ sodium carbonate solution, deionized water constant volume to $25 \mathrm{~mL}$, shake, prepare gallic acid mass concentration were $0,1.0,2.0,3.0,4.0,5.0,6.0$, $7.0 \mu \mathrm{g} / \mathrm{mL}, 30^{\circ} \mathrm{C}$ placed $1.5 \mathrm{~h}$, at the wavelength of $750 \mathrm{~nm}$ determination of absorbance, draw mass concentration-absorption curve ${ }^{[5]}$.

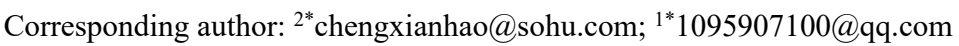




\subsection{Experimental treatment}

\subsubsection{Effect of ultrasonic time on polyphenol yield}

Reference Li et al. ${ }^{[6]}$ slightly changed. $1.0 \mathrm{~g}$ blueberry leaf powder was weighed, and the effects of extraction time on the yield of polyphenols from blueberry leaves were investigated at the solid-liquid ratio of $1: 25(\mathrm{~g} / \mathrm{mL})$, ethanol concentration of $60 \%$, and ultrasonic power of 250W.

\subsubsection{Effect of ethanol concentration on polyphenol yield}

The $1.00 \mathrm{~g}$ blueberry leaf powder was accurately weighed. Under the conditions of ultrasonic time of $6 \mathrm{~min}$, ultrasonic power of $250 \mathrm{~W}$, and solid-liquid ratio of 1:35 $(\mathrm{g} / \mathrm{mL})$, the effects of ethanol concentration of $20 \%, 40 \%$, $60 \%, 80 \%$, and $100 \%$ on the yield of polyphenols from blueberry leaves were investigated.

\subsubsection{Effect of solid-liquid ratio on polyphenol yield}

The $1.00 \mathrm{~g}$ blueberry leaf powder was accurately weighed. Under the conditions of ultrasonic time of $6 \mathrm{~min}$, ultrasonic power of $250 \mathrm{~W}$ and ethanol concentration of $60 \%$, the effects of solid-liquid ratio of 1:15, 1:25, 1:35, $1: 45$ and $1: 55(\mathrm{~g} / \mathrm{mL})$ on the yield of polyphenols from blueberry leaves were investigated.

\subsubsection{Determination of polyphenol content}

Folin phenol colorimetric method was used to determine the content of polyphenols. The method of $\mathrm{Li}^{[7]}$ was slightly modified. The regression curve equation of polyphenol absorbance and polyphenol concentration is $y=15.511 x-0.0032(R 2=0.9992)$, where $y$ is the absorbance of polyphenol at $752 \mathrm{~nm}$ and $\mathrm{x}$ is the concentration of polyphenol $(\mathrm{mg} / \mathrm{mL})$. The absorbance of polyphenols in blueberry leaves was determined at $752 \mathrm{~nm}$, the concentration of polyphenols was calculated according to the regression curve equation, and the yield of polyphenols was calculated according to the following formula.

Polyphenol yield $(\%)=C \times V \times n \times 100 / 1000 m$ (1)

Among them: $\mathrm{C}$ is the blueberry leaf polyphenol concentration $(\mathrm{mg} / \mathrm{mL}), \mathrm{V}$ is the extraction sample volume $(\mathrm{mL}), \mathrm{n}$ is the dilution ratio, $\mathrm{m}$ is the blueberry leaf mass (g).

\subsubsection{Nitrite removal ability}

It was slightly modified by referring to the method of Zhao ${ }^{[8]}$. Take three test tubes and number them $\mathrm{A}, \mathrm{B}$ and $\mathrm{C}$ respectively. Add $2 \mathrm{~mL}$ of extract to tube $\mathrm{A}, 2 \mathrm{~mL}$ of extract to tube $\mathrm{B}$, and $2 \mathrm{~mL}$ of deionized water to tube $\mathrm{C}$. Then $2 \mathrm{~mL} 10 \mu \mathrm{g} / \mathrm{mL} \mathrm{NaNO}{ }_{2}$ solution was added to tube $\mathrm{A}$ and tube $\mathrm{C}$, and $2 \mathrm{~mL}$ deionized water was added to tube $\mathrm{B}$. After shaking the three tubes separately, the tubes were left standing at room temperature for $5 \mathrm{~min}$. Then, $2 \mathrm{~mL} 0.4 \%$ p-aminobenzene sulfonate solution was added to test tubes
A, B and $\mathrm{C}$ respectively, and mixed. After standing for $15 \mathrm{~min}, \quad 1 \mathrm{~mL} \quad 0.2 \%$ naphthalene ethylenediamine hydrochloride solution was added to the three test tubes respectively, mixed, and stood for $15 \mathrm{~min}$ in the dark. The absorbance value was measured with a $2 \mathrm{~cm}$ colorimetric cup at the maximum absorption wavelength of $538 \mathrm{~nm}$. Taking deionized water as reference, the absorbance value $\mathrm{A}_{0}$ of $\mathrm{C}$ tube without extraction solution was measured, and the absorbance value $\mathrm{A}$ of $\mathrm{A}$ tube with extraction solution was measured with $\mathrm{B}$ tube as reference.

The following formula was used to calculate the nitrite clearance rate:

$$
S(\%)=\left(1-\frac{A_{i}-A_{j}}{A_{0}}\right) \times 100
$$

\subsubsection{Determination of $D P P H \bullet$ scavenging ability}

DPPH scavenging ability was modified according to the method of $\mathrm{Li}^{[9]}$. $0.2 \mathrm{mmol} / \mathrm{L}$ DPPH solution was prepared with anhydrous ethanol and kept away from light. The reagent was ready for use. Three test tubes were selected and labeled as A, B and C. First, $2 \mathrm{~mL}$ of the solution to be tested was added to each of $\mathrm{A}$ and $\mathrm{B}$, and $2 \mathrm{~mL}$ of anhydrous ethanol was added to C. Secondly, $2 \mathrm{~mL}$ DPPH solution was added to A and C respectively, and $2 \mathrm{~mL}$ deionized water was added to $\mathrm{B}$. Three test tubes were placed in the dark for 30 minutes, and the absorbance value was measured at $517 \mathrm{~nm}$.

$$
S(\%)=\left(1-\frac{A_{i}-A_{j}}{A_{0}}\right) \times 100
$$

Ai: Absorbance of liquid to be measured;

$\mathrm{A}_{\mathrm{j}}$ : Background value of liquid to be measured;

$\mathrm{A}_{0}$ : Control value of blank tube.

\section{Results and analysis}

\subsection{Effects of different ultrasonic time on the yield of polyphenols from blueberry leaves}

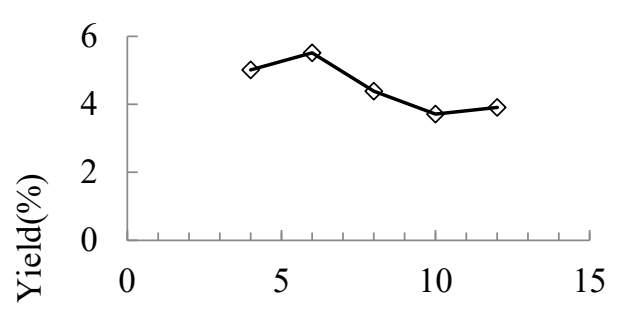

Time

Fig.1 Effects of different ultrasonic time on the yield of polyphenols from blueberry leaves

It can be seen from Fig.1 that the extraction rate of polyphenols increased first and then decreased with the ultrasonic time, and the optimum extraction time was $6 \mathrm{~min}$. 


\subsection{Effect of ethanol concentration on the yield of polyphenols from blueberry leaves}

It can be seen from Fig. 2 that the extraction efficiency is the highest when the concentration of ethanol is $60 \%$, and either the concentration is too high or too low, which is not conducive to extraction. According to the principle of similar phase solution, the polyphenols of blueberry leaves can be fully dissolved in the solvent with similar polarity so as to be extracted.

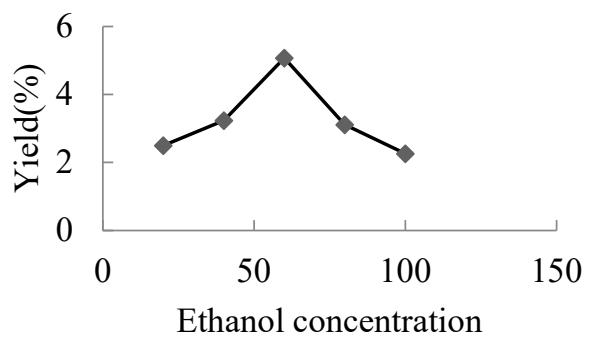

Fig.2 Effect of ethanol concentration on the yield of polyphenols from blueberry leaves

\subsection{Effect of material liquid ratio on the yield of polyphenols from blueberry leaves}

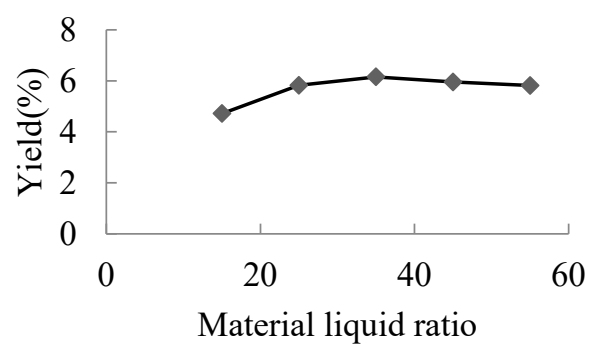

Fig.3 Effect of material liquid ratio on the yield of polyphenols from blueberry leaves

From Fig.3, solid-liquid ratio 1:35 had the highest extraction efficiency. too much ethanol was not conducive to the extraction of polyphenols, so the solid-liquid ratio was 1:35 is the best extraction ratio.

\section{4 orthogonal experiment}

Tab1. Results of orthogonal experiment of material liquid ratio, ethanol concentration(\%) and ultrasonic time(min)

\begin{tabular}{cccc}
\hline $\begin{array}{c}\text { material liquid } \\
\text { ratio }\end{array}$ & $\begin{array}{c}\text { ethanol } \\
\text { concentration } \\
(\%)\end{array}$ & $\begin{array}{c}\text { ultrasonic } \\
\text { time(min) }\end{array}$ & $\begin{array}{c}\text { the yield of } \\
\text { polyphenols }(\%)\end{array}$ \\
\hline $1: 25$ & 40 & 4.00 & 5.18 \\
$1: 25$ & 80 & 6.00 & 3.75 \\
$1: 25$ & 60 & 8.00 & 5.38 \\
$1: 35$ & 60 & 4.00 & 5.85 \\
$1: 35$ & 40 & 6.00 & 5.70 \\
$1: 35$ & 80 & 8.00 & 6.11 \\
$1: 45$ & 80 & 4.00 & 9.06 \\
$1: 45$ & 60 & 6.00 & 6.09 \\
$1: 45$ & 40 & 8.00 & 6.02 \\
\hline
\end{tabular}

According to the results of orthogonal experiment, the optimal extraction efficiency was obtained under the conditions of $80 \%$ ethanol concentration, 4min ultrasonic time and 1:45 solid-liquid ratio, which was different from the optimal extraction conditions of $60 \%$ ethanol concentration, 6min ultrasonic time and 1:35 solid-liquid ratio under the single factor condition, indicating the optimization of the experimental results by orthogonal experiment.

\subsection{Nitrite removal rate}

According to the Fig.4, the nitrite removal rate of blueberry leaves in May was the highest at the same concentration. For blueberry leaves in different months, the higher the concentration of extract, the higher the clearance rate.

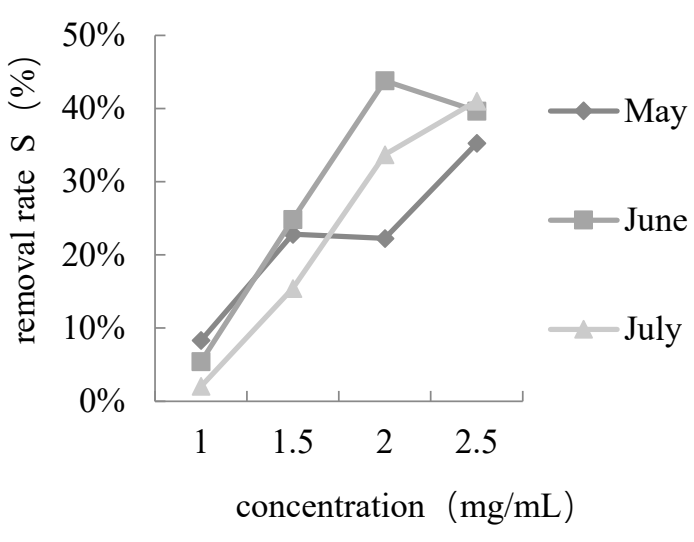

Fig.4 Nitrite removal rate of blueberry leaf extracts with different concentrations in May, June and July 


\subsection{DPPH scavenging rates}

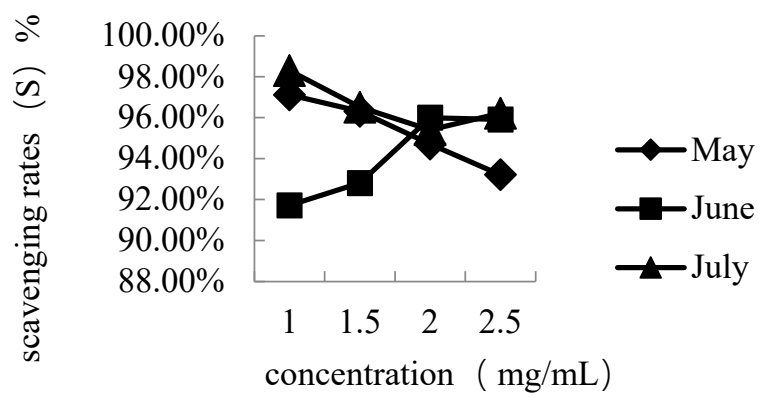

Fig.5 DPPH scavenging rates of blueberry leaf extracts with different concentrations in May, June and July According to the Fig.5, under the same concentration, the scavenging rate of DPPH of blueberry leaves in July was the highest. For blueberry leaves in different months, the lower the concentration of extract, the higher the scavenging rate.

\section{Conclusion}

Studies have shown that blueberry leaves contain relatively rich total phenols, and have strong free radical scavenging ability and reducing power ${ }^{[10]}$.The results of single factor experiment showed that the optimal extraction conditions existed regardless of ultrasonic time, ethanol concentration and solid-liquid ratio, and too high or too low would affect the extraction rate. The optimal extraction conditions of polyphenols from blueberry leaves were as follows, $9.06 \%$ with 4 min ultrasonic time, $80 \%$ ethanol concentration and 1:45 $(\mathrm{g} / \mathrm{mL})$ ratio. Ultrasonic assisted extraction is a convenient and high yield method, which is suitable for the extraction of polyphenols from blueberry leaves. However, the orthogonal experiment showed that the interaction of different extraction conditions was different from the accumulation of single factor optimal conditions.

\section{Acknowledgements}

Research on comprehensive utilization and pilot production of secondary fruit of Wudi jujube, provincial key R \& D plan in 2019 (tackling key scientific and technological problems for public welfare), (Project No. 2019GSF107083)

Research on the theory and practice of the deep integration course of specialty and innovation based on the cultivation of students' innovation and entrepreneurship--Taking bioengineering as an example(JG201918)

\section{Reference}

1. E L Zh, Z L, H R Zh. Study on nitrite scavenging capacity of sea buckthorn leaves [J]. Food industry science and technology, 2006(3):76-77.

2. G Y Y, B J Q, J Y, et al. Effects of blueberry varieties and extraction methods on the bioactivity of blueberry leaves[J]. Journal of Shanghai Jiaotong University: Agricultural Sciences, 2014, 32(1):49-52.

3. J F, X X Z, C Y L. Polyphenol vacuum extraction process optimization of blueberry leaves $[\mathrm{J}]$. Food industry technology, 2012, 033(016):315-318,371.

4. Takeshita, M, Ishida, Y.-i, Akamatsu, E,et al. Proanthocyanidin from Blueberry Leaves Suppresses Expression of Subgenomic Hepatitis C Virus RNA[J]. Journal of Biological Chemistry, 284(32):21165

5. Y C L, M H Zh, C Y M, et al. Optimization of ultrasonic-assisted extraction of polyphenols from blueberry leaves by response surface methodology $[\mathrm{J}]$. Food industry technology, 2015(15):236-240.

6. H W, Y L W, L Zh, et al. Research progress on extraction methods and functional activities of polyphenols from blueberries[J]. Food and machinery, 2015, 031(002):257-261,266.

7. B L, Y L, X J M, et al. Optimization of ultrasonic-assisted extraction of polyphenols from Lonicera edulis and its antioxidant activity by response surface methodology $[\mathrm{J}]$. Food industry science and technology, 2006(3):76-77.

8. EL Z, Z L, HL Z. Study on nitrite scavenging ability of seabuckthorn leaves[J]. Food industry science and technology, 2006 (3): 76-77.

9. G Y Y, B J Q, J Y, et al. Effects of blueberry varieties and extraction methods on bioactivity of blueberry leaves [J]. Journal of Shanghai Jiaotong University: Agricultural Science Edition, 2014, 32 (1): 49-52.

10. D L, T H M, H N S. Research progress on separation, purification and hypoglycemic activity of polyphenols from sweet potato leaves and stems [J]. Acta nucifera Sinica, 2021, 35 (02): 178-191. 